\title{
Proteccionismo en la industria automotriz colombiana
}

\author{
Carlos Ronderos / cert@etb.net.co \\ Universidad Sergio Arboleda \\ Colombia \\ Asistentes de investigación: \\ Javier Galán ${ }^{1}$ \\ María Clara Ávila \\ María Alejandra Niño
}

\section{RESUMEN:}

Este trabajo utiliza el concepto de protección efectiva para calcular los niveles de protección que han cobijado a la industria de ensamble automotriz en Colombia y valorar si esos niveles de protección han logrado los objetivos de una política de sustitución de importaciones que, mediante este mecanismo, busca consolidar una industria nacional de bienes de consumo durables con una creciente participación de insumos nacionales, permitiendo nuevas dinámicas de generación de inversión y empleo. Para ello se utilizan las cifras de la Encuesta Nacional Manufacturera de Colombia y los datos de incorporación de insumos nacionales que reportan las empresas ensambladoras establecidas en Colombia, y se revisan los cambios en las políticas y la normatividad que ha reglamentado esta actividad. Lo que se concluye del análisis que incorporan los elementos precedentes es que, contrario a los que cabría esperar, la participación de insumos nacionales ha decrecido de manera que el impacto sobre inversión y empleo resultan onerosos si se comparan con los sacrificios fiscales que implican los niveles de protección existentes. Finalmente, el artículo hace una reflexión sobre la conveniencia de perpetuar este tipo de políticas.

Palabras clave: protección efectiva, automotriz, costo de protección, sustitución de importaciones, ensamblaje automotriz, empleo, inversión

\section{ABSTRACT:}

This paper employs the concept of Effective Protection in order to calculate the real level of protection that has favored the automotive assembly industry in Colombia

\footnotetext{
${ }^{1}$ Profesor Investigador de la Escuela de Economía de la Universidad Sergio Arboleda, Bogotá, Colombia. Tuvo a su cargo el desarrollo matemático de los cálculos que se realizaron el presente trabajo.
}

ISSN 1541-8561 | 21 
and to value if this level of protection has helped to achieve the goals of an Import Substitution Policy. A policy that seeks to establish, through this protection mechanism a national industry of consumer durable goods, with an increasing share of domestic inputs generating alongside new dynamics in investment and employment generation. For this purpose, this paper works with data from the Colombian National Manufacture Survey (Encuesta Nacional Manufacturera de Colombia), data provided by the industry on incorporation of local inputs, and a review of changes in policy and legislation affecting the way in which this mechanism operates. Contrary to what would be expected, analysis shows that share of local inputs has decreased, bringing as a result that the cost of protection has been very high when compared to investment and employment generation. The paper concludes with some thoughts as to the convenience of maintaining this type of policy.

Keywords: effective protection, automotive, protection cost, import substitution, automotive assembly, employment, investment

\section{INTRODUCCIÓN}

El propósito del presente documento es calcular la protección efectiva de la cual ha gozado el sector de ensamblaje automotriz a la luz de la normatividad colombiana y andina vigente, valorando el impacto que esta protección tiene sobre el empleo y la inversión, así como el costo que representa para la sociedad sostener estos niveles de empleo. Para ello, se reseña en la próxima sección la normatividad y las políticas que han regulado la protección de la industria de ensamble, así como los fundamentos teóricos sobre los cuales descansa esa política. Posteriormente, se revisa la metodología que permite el cálculo de la protección efectiva, teniendo en cuenta la manera como diversos autores abordan el tema para continuar, en la sección cuarta de este trabajo, con un análisis de los datos que permiten calcular la protección efectiva y el impacto de esta política sobre las variables objetivo (empleo e inversión). Finalmente, se formulan conclusiones y reflexiones que buscan orientar la política pública al evaluar la permanencia de este tipo de modelos de desarrollo industrial. 


\section{NORMATIVIDAD AUTOMOTRIZ ANDINA}

El sistema de protección de la industria automotriz colombiana tiene su origen en las políticas promulgadas por la Comisión Económica para América Latina y el Caribe (CEPAL) para impulsar los procesos de industrialización (Prebisch, 1964) que se fundamentan en la imposición de altos aranceles a los bienes finales y aranceles bajos, o inexistentes, para los insumos que se utilicen en la producción de ese bien (Echavarría et al., 2006). Se pensaba que este mecanismo arancelario permitiría la creación de una industria nacional que no solo era indispensable para el desarrollo económico de la región sino que también constituía la estrategia para superar la creciente brecha que los separaba de los países ricos, revirtiendo un persistente deterioro en los términos de intercambio entre países pobres que vendían materias primas y países ricos que vendían bienes manufacturados.

Amparados por este sistema de protección, algunas compañías automotrices instalaron plantas ensambladoras de vehículos destinadas a atender los mercados locales en diferentes países de América Latina. En el caso de Colombia, las compañías multinacionales que se establecieron fueron la americana General Motors (GM Colmotores 1956), la francesa Renault (Sofasa 1969) y la japonesa Mazda (CCA 1982). Los mecanismos de protección del sector automotriz se han dado en el marco de la integración andina que sufrió una modificación en 1993 con el Convenio de Complementación en el Sector Automotor, el cual fue ampliado en 1994 y actualizado en 1999. En virtud de estos acuerdos, se estableció un arancel externo común del 35\% para los "vehículos para el transporte de pasajeros hasta de 16 personas incluido el conductor; y los vehículos de transporte de mercancías de un peso total con carga máxima inferior o igual a 4,537 toneladas (o 10,000 libras americanas), así como sus chasis cabinados" (1A) (Acuerdo 323 CAN). Lo anterior permite el comercio de dichos vehículos sin arancel entre los países signatarios del acuerdo (Colombia, Venezuela y Ecuador) y exime de arancel la importación de los insumos utilizados para en ensamblaje de estos vehículos, insumos que se conocen como material CKD. Este 
convenio que tenía una vigencia de diez años proponía un nivel de integración regional del $50 \%$ que, once años después, no se ha cumplido. En el transcurso de la vigencia del convenio, Venezuela se retiró de la Comunidad Andina y, por tanto, del convenio. Ecuador lo denunció, de tal forma que el único país del convenio que mantiene el sistema es Colombia.

Es importante señalar que el convenio vigente representa un retroceso significativo en materia de incorporación de insumos nacionales, si se compara con la normatividad de la década de los noventa. Como se puede apreciar en la Tabla 1, el porcentaje de integración regional exigido para dar origen en el ensamblaje de los vehículos categoría 1 ha disminuido, pasando de un $40 \%$ en 1994 a un 24,8\% en $2000^{2}$. Mientras en la década de los noventa la economía colombiana adoptó un modelo de apertura, el sector automotriz incrementó sus niveles de protección, ya que a medida que disminuye el contenido regional e incrementa el importado (sin pago de aranceles), aumenta la tasa de protección efectiva, como se demostrará en este trabajo.

Tabla 1

Porcentaje de integración regional en ensamblaje de vehículos categoría 1 en diferentes fechas

\begin{tabular}{|l|c|c|}
\hline \multicolumn{1}{|c|}{ NORMA } & FECHA & \% DE INTEGRACIÓN \\
\hline Resolución 354 (Andina) & 1994 & $40 \%$ \\
\hline Decreto 440 (Colombia) & 1995 & $33 \%$ \\
\hline Resolución 323 (Andina) & 2000 & $24,8 \%$ \\
\hline
\end{tabular}

Fuente: Elaboración propia.

La normatividad sobre régimen automotor, igualmente, se ocupa del porcentaje de integración que deben tener las autopartes que entran al proceso de ensamblaje para cumplir con el requisito de origen. Desde comienzos de la década de los 90, para que un insumo utilizado en el ensamblaje automotriz sea considerado "nacional", debe ser manufacturado con partes y piezas nacionales cuyo valor

\footnotetext{
${ }^{2}$ Este porcentaje se incrementa en el tiempo como puede apreciarse en la Tabla 2.

$24 \quad$ ISSN 1541-8561

FORUM EMPRESARIAL VOL. 19 NÚM. 1 • VERANO 2014
} 
sea, como mínimo, el $45 \%$ del valor total. Lo anterior significa que lo que se considera originario en realidad contiene hasta un $55 \%$ no originario. En resumen, las partes "nacionales" que se ensamblan pueden ser hasta en un 55\% no originaria, y el vehículo que contiene esas partes es originario si cumple con los requisitos de la Resolución Andina 323 (ver Tabla 2), goza de la exención arancelaria y tiene acceso a los mercados andinos sin barreras arancelarias.

La fórmula que la normatividad andina estableció para el cálculo del porcentaje de integración subregional es:

(1) $I S=\frac{M O}{M O+M N O} \times 100$

Donde:

IS = Integración Subregional

$\mathrm{MO}=$ Material Originario

MNO = Material No Originario de la Subregión (CKD)

Esta normatividad, igualmente, estableció el porcentaje de Integración Subregional que rige en cada año del convenio para cada país, siendo los porcentajes para Colombia los que aparecen en la Tabla 2. Además, extendió el régimen a las autopartes que constituyen el material original (MO) que integran las ensambladoras.

Tabla 2

Porcentajes mínimos de integración categoría 1

\begin{tabular}{|c|r|c|r|}
\hline AÑ & \% DE IS & AÑ & \% DE IS \\
\hline 2000 & 24.8 & 2005 & 30.4 \\
\hline 2001 & 25.8 & 2006 & 31.5 \\
\hline 2002 & 26.8 & 2007 & 32.6 \\
\hline 2003 & 27.8 & 2008 & 33.7 \\
\hline 2004 & 28.8 & 2009 & 34.6 \\
\hline
\end{tabular}

Fuente: Resolución 323 CAN.

Dado que no ha habido renovación del convenio y que, como ya se anotó, ni Venezuela ni Ecuador han mostrado voluntad en avanzar en el tema, Colombia sigue midiendo el nivel de integración con los porcentajes de 2009. 


\section{Metodología}

En esta sección del trabajo se revisan las fuentes de los datos que se utilizan, así como los elementos teóricos que permiten el cálculo de la protección nominal y la protección efectiva.

\section{Datos utilizados}

Las empresas CCA (Mazda), Sofasa (Renault) y GM Colmotores (General Motors) ensamblan en Colombia la totalidad de los vehículos de la categoría $1^{\underline{a}}$ (hasta 16 pasajeros). En la Tabla 3 se recogen los porcentajes de integración del material originario que estas compañías han reportado al Ministerios de Comercio Industria y Turismo.

Tabla 3

Contenido de material originario en los procesos de ensamble categoría 1 según reportes de las ensambladoras al MICT

\begin{tabular}{|l|l|r|r|c|}
\hline CIIU 341 & CATEGORÍA & \multicolumn{1}{c|}{$\begin{array}{c}\text { MATERIAL } \\
\text { ORIGINARIO }\end{array}$} & $\begin{array}{c}\text { MATERIAL NO } \\
\text { ORIGINARIO }\end{array}$ & $\begin{array}{l}\text { INTEGRACIÓN } \\
\text { SUBREGIONAL }\end{array}$ \\
\hline 2002 & Categoría 1 & 393.842 .351 .711 & 954.948 .974 .007 & $29,20 \%$ \\
\hline 2003 & Categoría 1 & 378.076 .802 .979 & 904.643 .856 .420 & $29,47 \%$ \\
\hline 2004 & Categoría 1 & 623.536 .284 .074 & 1.414 .196 .335 .181 & $30,60 \%$ \\
\hline 2005 & Categoría 1 & 760.986 .759 .786 & 1.543 .856 .811 .642 & $33,02 \%$ \\
\hline 2006 & Categoría 1 & 914.820 .738 .035 & 1.875 .480 .401 .151 & $32,79 \%$ \\
\hline 2007 & Categoría 1 & 1.181 .533 .526 .737 & 2.279 .977 .539 .505 & $34,13 \%$ \\
\hline 2008 & Categoría 1 & 644.240 .865 .149 & 1.209 .995 .919 .807 & $34,74 \%$ \\
\hline 2009 & Categoría 1 & 407.932 .097 .700 & 757.176 .290 .147 & $35,01 \%$ \\
\hline
\end{tabular}

Fuente: Ministerio de Comercio, Industria y Turismo de Colombia.

Como puede observarse, la industria cumple de manera holgada en todos los años con los requisitos de integración obteniendo origen para los vehículos ensamblados en Colombia y permitiendo a las ensambladoras importar al país el material No Originario sin el pago de arancel o impuesto al valor agregado.

Se trata de una industria pequeña que ensambla alrededor de cien mil vehículos por años en esta categoría, como puede observarse en la Tabla 4: 
Tabla 4

Ensamble de vehículos categoría 1

\begin{tabular}{|r|r|r|r|}
\hline AÑO & $\begin{array}{c}\text { UNIDADES } \\
\text { PRODUCIDAS }\end{array}$ & $\begin{array}{c}\text { UNIDADES } \\
\text { VENDIDAS }\end{array}$ & $\begin{array}{c}\text { UNIDADES EX- } \\
\text { PORTADAS }\end{array}$ \\
\hline 2002 & 72.650 & 50.928 & 20.969 \\
\hline 2003 & 54.705 & 54.926 & 8.515 \\
\hline 2004 & 85.457 & 57.248 & 27.487 \\
\hline 2005 & 99.149 & 58.168 & 40.662 \\
\hline 2006 & 126.274 & 78.667 & 67.317 \\
\hline 2007 & 168.143 & 95.080 & 5.770 \\
\hline 2008 & 97.179 & 76.298 & \\
\hline 2009 & 45.902 & & \\
\hline
\end{tabular}

Fuente: DANE, ACOLFA.

Se complementa esta información con los datos que suministra la Encuesta Nacional Manufacturera (ENM) en las calificaciones CIIU 341 de ensamblaje y 334 de autopartes que publica el Departamento Nacional de Estadísticas (DANE). El período de análisis comprende de 2002 a 2008, ya que se concluyó que las cifras de 2009, que también están disponibles en la ENM, no son consistentes (ver Anexo $5)$.

Como ya se señaló, en la industria de ensamblaje se debe diferenciar el valor agregado de las ensambladoras y aquel de la industria de autopartes. Las ensambladoras importan material CKD y usan material de integración regional (CAN) como insumos para el proceso de armado del vehículo terminado. Las empresas de autopartes utilizan materiales regionales ${ }^{3}$ e importados para la elaboración de las piezas que más adelante serán integradas al vehículo terminado como material originario.

Para determinar la proporción del valor agregado nacional es necesario calcular la porción real de origen nacional en las autopartes,

${ }^{3}$ El material regional incluye material proveniente de los países de CAN que en cada año pertenecían al convenio automotriz (Venezuela y Ecuador), pero dado que no se discrimina en las estadísticas qué proporción de este material originario de carácter regional proviene de Colombia y qué proporción de los otros países miembros del convenio se toma todo como "nacional", para el cálculo de la protección efectiva, lo que subestima el grado de protección a la industria nacional. 
según lo establecido en la Resolución 323 de la CAN ya citada. Para realizar el cálculo de la protección efectiva (ver consideraciones teóricas), es necesario determinar los porcentajes del material verdaderamente originario. Para ello se resta del material originario-MO- la porción de éste que no es originario. Los cálculos se presentan en el Anexo 1.

Los datos de la EAM no discriminan los componentes de producción bruta por categorías de vehículos y, por ello, para calcular la producción bruta de la categoría 1 se utiliza la siguiente fórmula:

(2) $P B_{I t}=C K D_{I t}+M O_{I t}+\left(P B_{I t} * V A_{t}\right)$

Donde:

$P B_{I t}=$ Producción bruta de la categoría 1 en el año t (Fuente Mincomercio reporte ensambladoras).

$C K D_{I t}=$ CKD o Material no Originario tomado del reporte de las ensambladoras de vehículos para la categoría 1 en el año t.

$M O_{I t}=$ Material Originario tomado del reporte de las ensambladoras de vehículos para la categoría 1 en el año t.

$V A_{t}=$ El porcentaje de valor agregado (CIIU 341) de la Encuesta Anual Manufacturera (EAM) en el año t.

Para que la serie sea comparable a lo largo del período de estudio, se llevaron los valores de la producción bruta, consumo intermedio y valor agregado a términos constantes, utilizando para ello el Índice de Precios al Productor (IPP) publicado por el DANE para las cifras de producción doméstica, y el IPP que publica la Oficina de Estadísticas Laborales de los Estados Unidos para las cifras de los bienes importados. Durante el período de estudio, el peso colombiano sufrió una fuerte revaluación y, dado que las fórmulas para el cálculo de contenido regional comparan producción nacional en pesos con CKD (Material No Originario) en pesos a la tasa de cada año, se distorsiona la real participación de bienes de origen nacional en el total de la producción. Por ello se realizó un ajuste para eliminar el efecto cambiario sobre los valores en pesos de los bienes de origen extranjero (ver Anexo 2). 


\section{MARCO TEÓRICO}

En esta sección se analizan las fórmulas propuestas por diferentes autores para el cálculo tanto de la protección nominal como de la protección efectiva.

\section{Protección nominal}

La protección nominal se puede establecer de una forma directa, tomando la diferencia entre el arancel a las importaciones de los bienes finales y el impuesto a las importaciones de los bienes intermedios. Es decir:

(3) $n_{j}=t_{j}-\sum_{i=1}^{n} a_{i j} t_{i}$

Donde:

$n_{j}=$ Es La protección nominal.

$t_{j}=\mathrm{El}$ arancel del bien final.

$a_{i j}=$ Los porcentajes de participación de los bienes intermedios.

$t_{i}=$ Los aranceles de los bienes intermedios.

Si el arancel aplicado al bien final es igual al arancel aplicado a bienes intermedios, la protección nominal será mayor a 0 , pero menor al arancel del bien final. Si el arancel de los bienes intermedios es mayor a la del bien final, el arancel nominal será bajo, e incluso negativo, y en la medida en que el arancel de los bienes intermedios sea menor al del bien final (que es normalmente el caso de procesos de protección que tienen como propósito la promoción de la industrialización), el arancel nominal se reducirá en menor proporción. En caso que el arancel de los bienes intermedios sea cero, el arancel nominal será igual al aplicado.

La protección nominal mide el cambio en el precio final del bien producido por la industria y su valor dependerá de la participación de los bienes intermedios en el proceso de producción.

\section{Protección efectiva}

La teoría de la protección efectiva establece la protección que un determinado arancel tiene sobre el valor agregado y no, como en 
el caso de la protección nominal, el efecto sobre el precio final del bien.

El precio del producto final de una industria i, se puede definir como (Basevi, 1966):

(4) $\quad 1=v_{j}+\sum_{i=1}^{n} a_{i j}$

Donde:

$v_{j}=$ Valor agregado en la industria $\mathrm{j}$, por dólar del producto final de la industria $\mathrm{j}$, en ausencia de impuestos a las importaciones.

$a_{i j}=$ Valor del insumo i, por dólar del producto final $\mathrm{j}$, en ausencia de impuestos a las importaciones de bienes intermedios.

Si los bienes finales de la industria están sujetos a impuestos a las importaciones, su valor se define como:

$$
1+t_{j}=v_{j}^{\prime}+\sum_{i=1}^{n} a_{i j}
$$

Donde:

$t_{j}=$ Impuestos a las importaciones del bien final de la industria j.

$v_{j}^{1}=$ Valor agregado en la industria j, por dólar del bien final de la industria $\mathrm{j}$.

$a_{i j}=$ Valor del insumo i, por dólar del bien final de la industriaj, en ausencia de impuestos a las importaciones de bienes intermedios.

Como los bienes intermedios no están sujetos a impuestos, entonces un arancel sobre el bien final debería tener como efecto un aumento del valor agregado por la industria local.

$$
v_{j}^{\prime}=\left(1+t_{j}\right)-\sum_{i=1}^{n} a_{i j}
$$


Introduciendo impuestos a los bienes intermedios, tenemos:

$$
v_{j}^{\prime}=\left(1+t_{j}\right)-\sum_{i=1}^{n} a_{i j}\left(1+t_{i}\right)
$$

De forma tal que el efecto que tienen los impuestos a las importaciones de bienes intermedios es reducir el valor agregado de la industria. Lo que así muestra Basevi es el fundamento teórico para el diseño de una política de sustitución de importaciones.

Grubel \& Johnson (1967), Basevi (1966), Cohen (1971), Alavi (1996) y Balassa (1965) han propuesto fórmulas de medición de la protección efectiva que pueden resumirse de la siguiente manera:

$$
f_{j}=\frac{\boldsymbol{v}_{j}^{\prime}-\boldsymbol{v}_{j}}{\boldsymbol{v}_{j}}
$$

El propósito de imponer un arancel alto al bien final, manteniendo un bajo arancel a bienes intermedios, es que se incentive el incremento del valor agregado doméstico, que es lo que se conoce como efecto subsidio. Si se incrementa el arancel sobre los insumos requeridos por la industria, se disminuye la protección efectiva sobre el bien final en lo que se conoce como efecto impuesto.

De acuerdo con Basevi (1966), la protección máxima está dada por la protección efectiva en ausencia de aranceles sobre los bienes intermedios y se compara con la protección efectiva que resulta del cálculo de esta protección en presencia de aranceles sobre los bienes intermedios.

$$
f_{j}=\frac{t_{j}-\sum_{i=1}^{n} a_{i j} t_{i}}{v_{j}}
$$

La tasa efectiva de protección también puede entenderse como: “[...] máxima proporción por la que el valor agregado por unidad de producto de una industria local excede el valor agregado por unidad de producto de una industria competitiva en el mercado mundial" (Basevi, 1966).

En consecuencia, si no existen impuestos a las importaciones de los bienes intermedios, la máxima protección será la relación entre el impuesto a las importaciones de los productos finales y el valor agregado por la industria.

ISSN 1541-8561 | 31 


$$
f_{j}=\frac{\left(1+t_{j}\right)-1}{v_{j}}=\frac{t_{j}}{v_{j}}
$$

La tasa efectiva de protección, también, se define como la diferencia entre el valor agregado en el país local, debido a la imposición de medidas proteccionistas tanto al bien final como a los insumos, sobre el valor agregado de libre comercio (Alavi, 1996 y Cohen, 1971).

$$
E R P=\frac{\left(V A_{D}-V A_{W}\right)}{V A_{W}} \times 100
$$

$V A_{d}=$ valor agregado a precios del país local.

$V A_{W}=$ valor agregado a precios de libre comercio o precios mundiales.

El valor agregado a precios del país local se obtiene:

$$
V A_{D}=\sum Q_{d p}-\sum Q_{d i}
$$

Donde:

$\Sigma Q_{d p}=$ Valor de la producción bruta, a precios del país local $\Sigma Q_{d i}=$ Valor total de los bienes intermedios, a precios del país local.

El valor agregado a precios de libre comercio o precios mundiales, se obtiene:

$$
V A_{W}=\sum Q_{w p}-\sum Q_{w i}
$$

Donde:

$\Sigma Q_{w p}=$ Valor de la producción bruta, a precios de libre comercio.

$\Sigma Q_{w i}=$ Valor total de los bienes intermedios, a precios de libre comercio.

Si ERP $>0$, la industria se encuentra protegida.

Si $\mathrm{ERP}<0$, no necesariamente implica que no exista protección. 
Existen dos formas de interpretar un valor negativo de la ERP: la primera, si el valor agregado a precios de libre comercio es mayor que el valor agregado a precios del país local. En este caso, la industria no se encuentra protegida.

Otra interpretación es que si el valor agregado a precios de libre comercio es cero o negativo, existe una alta tasa de protección, es decir, que si el bien final se va a vender a precios de libre comercio, el productor del país local estaría incurriendo en pérdidas. En esta interpretación una ERP negativa implica que existen marcadas diferencias en la función de producción entre países, es decir, la transformación de los insumos en el producto final es ineficiente en el país local comparado con las industrias del resto del mundo.

En entrevista realizada en mayo de 2011 con el Director de Investigación de Desarrollo Económico de la OMC, Patrick Low (Doctor en Economía de la Universidad de Sussex y Economista de la Universidad de Kent), obtuvimos una segunda aproximación para determinar la protección efectiva:

$$
f_{j}=\frac{\left(1+t_{j}\right)-v_{j}}{v_{j}}
$$

La protección efectiva mide el efecto que tiene sobre la industria la imposición de un arancel sobre el bien final, en ausencia de impuestos a los insumos.

\section{Resultados}

Para determinar la protección nominal y efectiva, se utilizaron las fuentes de datos ya mencionadas con los ajustes que aparecen detallados en los anexos y la metodología propuesta por Basevi (1966) (el Anexo 3 presenta los resultados obtenidos para cada año). Tanto los cálculos de la protección nominal como los de la protección efectiva se hacen para los vehículos de la categoría 1. Con la información obtenida, en los Anexos 1 al 4, se procedió a calcular la protección nominal y la protección efectiva, de acuerdo con las metodologías propuestas. Posteriormente se presenta el comportamiento que ha tenido el empleo y la formación bruta de capital en el sector para el 
período 2002-2008, con el fin de relacionar el grado de protección con estas variables.

\section{Protección Nominal}

En la Figura 1 se muestra la protección nominal utilizando la formula expuesta por Basevi.

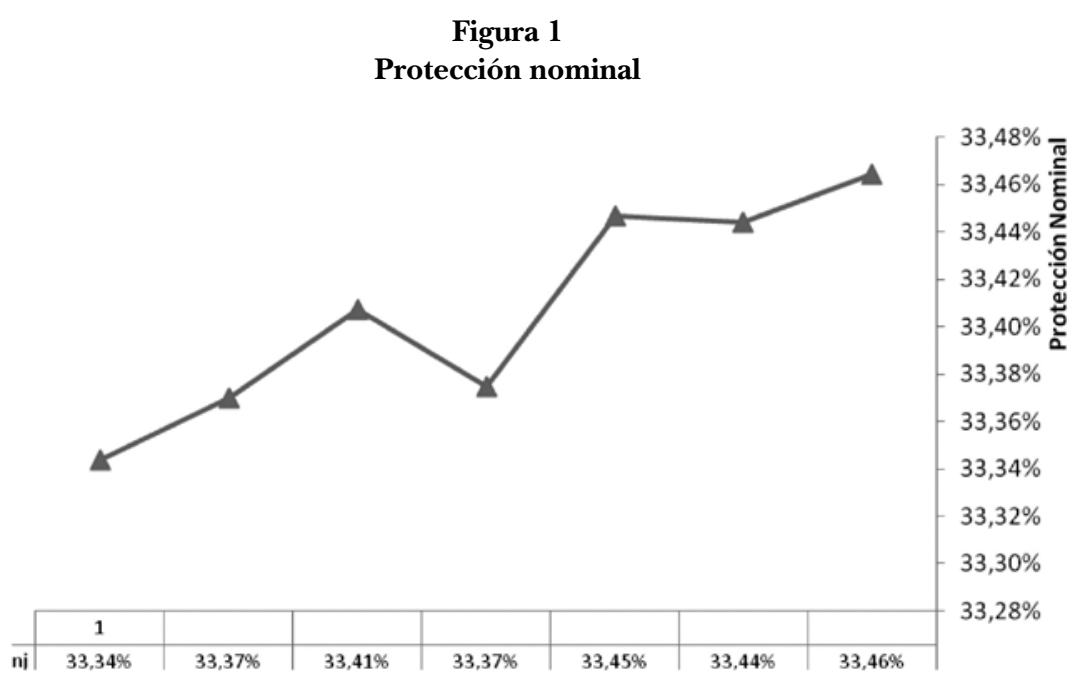

La protección nominal del sector automotriz colombiano ha oscilado entre el $33.34 \%$ y el $33.46 \%$, teniendo en cuenta que las materias primas importadas tienen, en promedio, un arancel del $2 \% 4$. Cuando no pesa arancel sobre las materias primas, la protección nominal será igual al arancel de las importaciones del bien terminado.

El resultado del cálculo corrobora lo expuesto por Basevi, en el sentido de que si el arancel a las importaciones de los bienes finales es mayor que el arancel a los bienes intermedios, la protección nominal será una tasa intermedia entre los dos aranceles.

\footnotetext{
${ }^{4}$ Para estimar este impuesto se tomaron los insumos asociados a la fabricación de vehículos del reporte "Materias primas, materiales y empaques consumidos y comprados, según tipo de artículo" elaborado por el DANE. 


\section{Protección efectiva}

Es importante resaltar que la industria de ensamble, y aquella que se genera en las autopartes como resultado de la demanda que realizan las ensambladoras, generan una dinámica en la industria nacional que no se limita a la proporción de material originario incorporado (porcentaje que mide la normatividad andina y que se ha depurado para este trabajo en el Anexo 1, teniendo en cuenta los REOS). Esta industria también genera una dinámica económica que se refleja en el valor agregado que se produce en el proceso de ensamblaje por concepto de los salarios que paga la industria, el uso de energía, la utilización de una infraestructura inmobiliaria y el pago de servicios de toda índole, entre muchos otros elementos que componen lo que la ENM registra como VALOR AGREGADO. Es por ello que, en el cálculo de la protección efectiva la diversas fórmulas que proponen los autores que se citan, al igual que en el cálculo de la fórmula de Basevi que se utiliza en esta sección, la protección efectiva mide tanto la incorporación de insumos como el valor agregado por la industria, porque solo así se puede valorar en su verdadera dimensión el efecto que tiene la protección sobre la economía local.

\section{Protección efectiva nORMatividad 1994-2008}

Utilizando la metodología de Basevi, los porcentajes de integración de material originario que ha establecido la normatividad, según aparece en la Tabla 1, y el porcentaje de valor agregado para cada uno de los años en que estuvo vigente la normatividad mencionada, según cifras de la ENM del DANE y el arancel nominal publicado $(35 \%)$, se calculó la tendencia de la protección efectiva entre los años 1994 y 2000 como aparece en la Figura 2.

Como era previsible, la disminución en el contenido regional requerido da lugar a un incremento en la protección efectiva. Es oportuno aclarar que este cálculo no se basa en datos de incorporación de insumo regional de las ensambladoras, sino en los porcentajes que exigía la norma, y es probable que la protección efectiva durante el período haya sido menor si la incorporación, que efectivamente realizaron las ensambladoras, fue mayor. No obstante lo anterior, lo que resulta interesante es que mientras en la década de los noventa 
Figura 2

Protección efectiva normatividad 19945-2000

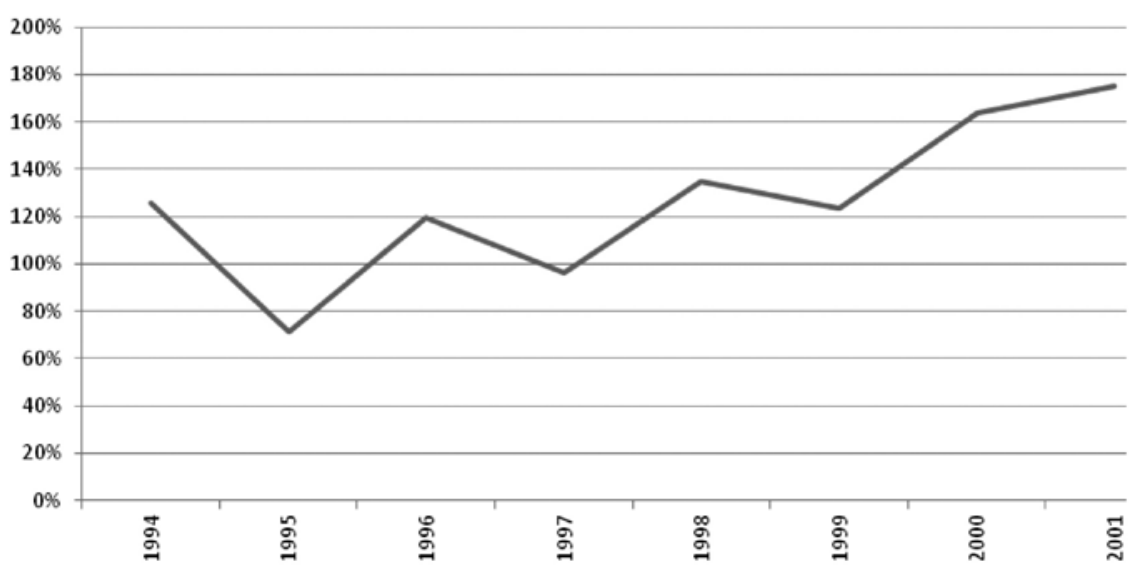

Fuente: DANE (Encuesta Anual Manufacturera) - Cálculos propios.

el país sufrió un apertura generalizada asociada a una disminución de aranceles, la industria de ensamble de vehículos gozó de una normatividad que le permitía una mayor protección, como lo refleja la figura anterior en la cual se observan niveles de Protección efectiva normatividad 1994-2008 que crecen desde una cifra cercana al $80 \%$ en 1995 a una cifra cercana al 180\% en el año 2000.

\section{Protección efectiva datos 2002-2008}

El cálculo de la protección efectiva que se realiza en esta sección se basa en la información sobre incorporación de Material Originario efectivamente reportado por las ensambladoras, el valor agregado reportado por la Encuesta Nacional Manufacturera y el arancel nominal que resultó para el período que parece descrito en la Gráfica 1. Estos cálculos se grafican en la Figura 3, en la cual la diferencia entre protección máxima que aparece en la línea roja y la que se de-

\footnotetext{
5 Para calcular la protección efectiva para 1994-2001, período en el cual no se cuenta con información detallada, se tomó como valor de la variable $\mathrm{v}_{\mathrm{j}}$ el valor reportado en la Encuesta Anual Manufacturera como Valor Agregado. 
nomina Basevi está dada por la diferencia entre el arancel nominal publicado $(35 \%)$ y el que resulta del cálculo descrito en la Gráfica 1.

Figura 3

Protección efectiva (Basevi)

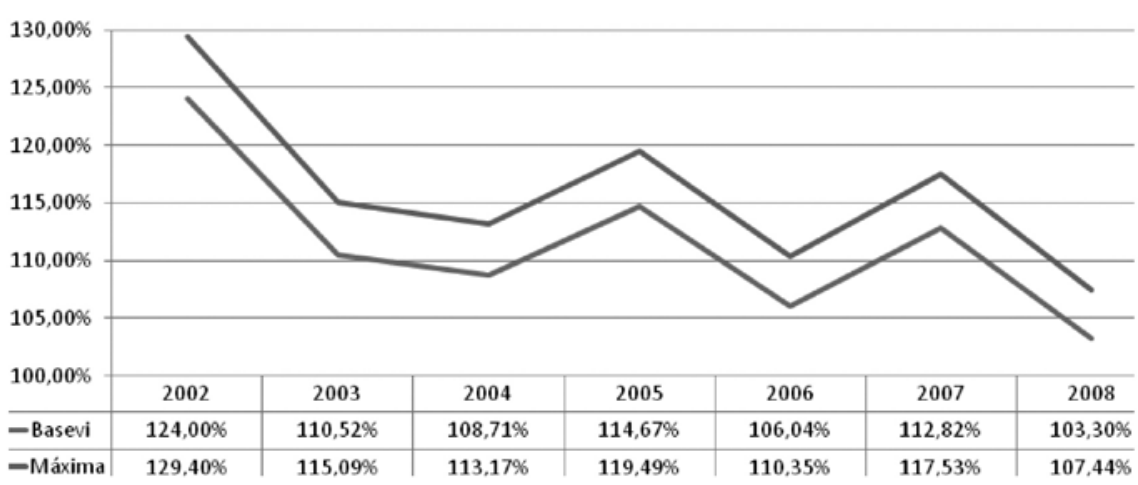

Fuente: Datos de Protección nominal Figura1, Mincomercio, EN-DANE, cálculos propios.

Los resultados de la metodología propuesta por Basevi indican que la protección efectiva del sector oscila entre el $124.00 \%$ y el $103.30 \%$, y se encuentra muy cerca de los valores máximos esperados. Como puede apreciarse, los niveles de protección efectiva que arrojan los cálculos para el período 2002-2008 son inferiores a los que arroja el cálculo sobre la normatividad. Ello se explica por dos razones: en primera instancia, a partir de 2002 el DANE incluye como valor agregado nacional las partes originarias de la subregión dentro del cálculo de producción nacional, lo cual hace que aparezcan como nacionales partes producidas en Venezuela y Ecuador, y que se refleje en una menor protección efectiva. La segunda razón puede encontrarse en el hecho de que las cifras de esta segunda serie son las cifras reales reportadas por las ensambladoras que, como puede observarse en la Tabla 3, son superiores a aquellas que ordena la normatividad andina. En consecuencia, las dos series no son comparables pero ambas reflejan altos niveles de protección que fueron crecientes en la década de los noventas y que se han mantenido a lo 
largo del presente siglo a niveles superiores al 100\%, que son niveles de protección muy por encima de la protección de la industria en otros países y una carga importante para los consumidores.

Con el fin de corroborar los resultados obtenidos por medio de la aplicación de la fórmula de Basevi, se procesaron los datos con la metodología propuesta por Alavi, la cual aparece detallada en la sección de teoría, y se encontraron altos niveles de protección negativos que indicarían que la industria no sería competitiva en ausencia de la protección, lo que resulta evidente al analizar los precios de venta al público de los vehículo comparados con aquellos de los países donde no hay protección.

\section{EFECTO SOBRE EL EMPLEO}

Para analizar los efectos de la protección sobre el empleo, se utilizó la información reportada por la Encuesta Anual ManufactureraDANE para el período de estudio. En primera instancia, se recogió la información sobre empleo permanente de las ensambladoras, dado que este dato sería el que más se asimila a empleo formal y, por lo tanto, a empleo de calidad.

La Figura 4 muestra la evolución del empleo permanente para el sector industrial de fabricación de vehículos automotores y sus motores (CIIU 341), y ésta refleja la tendencia decreciente que indicaría que las empresas ensambladoras no están siendo generadoras de nuevos empleos de calidad, a pesar de los altos niveles de protección de los cuales goza.

Existe un repunte en 2007 que no logra modificar la tendencia negativa, que refleja cómo de 2,103 trabajadores que había con empleo permanente en 2002 se pasó a 1,896 en 2008. El máximo número de personas contratadas en forma permanente por el sector de ensamble de vehículos se alcanzó en 2002. Las cifras de empleo permanente en las ensambladoras tienen dos inconvenientes si se utilizan como indicadores de impacto de la industria en el empleo. Primero, durante este período en Colombia existió una tendencia a desvincular trabajadores con empleos permanentes y remplazarlos por empleos temporales (Farné 2008), lo que ha generado una polémica que repercutió en la discusión de la aprobación del Tratado 
Figura 4

Personal remunerado permanentemente

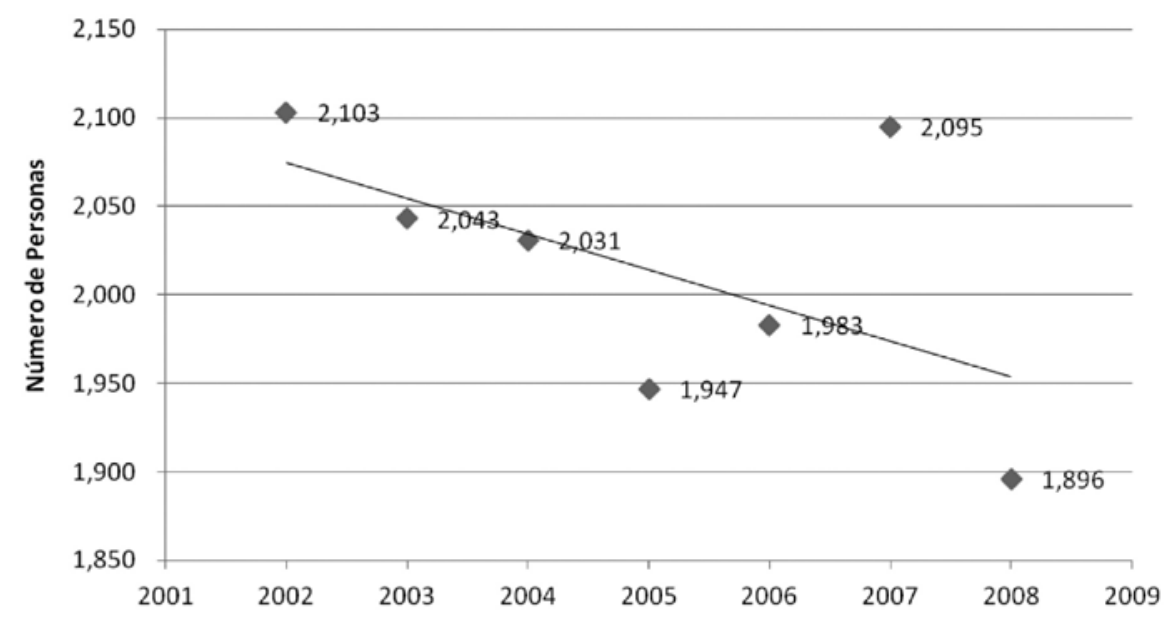

Fuente: DANE (EAM).

de Libre Comercio (TLC) con los Estados Unidos. Las cifras, en efecto, reflejan un número creciente de trabajadores temporales en la industria de ensamble. En segunda medida, es necesario incluir en este cálculo los empleos que genera la industria como resultado de la demanda de autopartes (MO) que incorpora en el proceso de ensamble. No se incluye el empleo generado por estas empresas en la venta, postventa y mantenimientos de vehículos, ya que estos empleos se generan de igual manera con vehículos importados o ensamblados localmente. De hecho, las empresas ensambladoras son importantes importadoras de vehículos, y en las salas de venta y en los talleres de reparación y mantenimiento se atiende de igual manera a los vehículos que estas empresas ensamblan y los que importan.

Las anteriores consideraciones llevaron a observar la tendencia de generación de empleo de la industria de ensamble teniendo en cuenta empleos temporales y los empleos que genera aquella proporción de la industria de autopartes que se destina al ensamble de 
vehículos ${ }^{6}$, dado que la industria de autopartes también genera empleos como resultado de la producción de autopartes para el mercado de reposición y para el mercado de exportación. Los resultados se presentan en la Figura 5.

Figura 5

Total personal remunerado

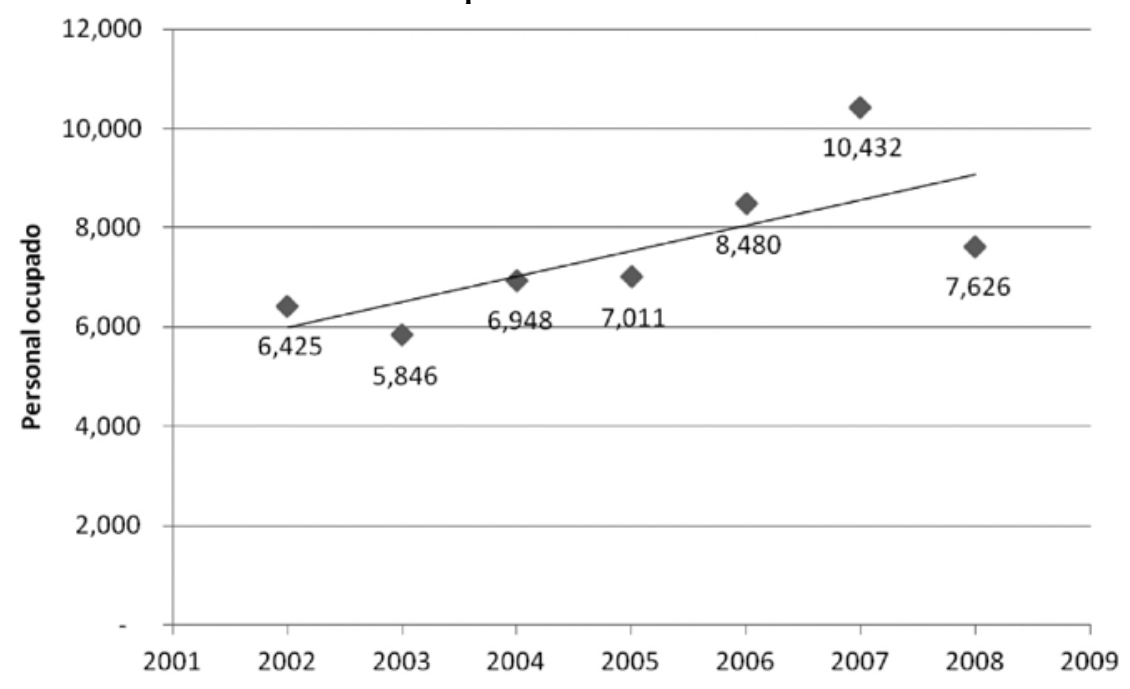

Fuente: DANE (EAM), ACOLFA (Manual estadístico 30), cálculos propios.

El total del personal ocupado presenta una tendencia positiva, con 6,425 trabajadores empleados en 2002 y 7,626 personas en 2008. Se observa una caída en el empleo en 2003 (-579 personas), seguida por un repunte acentuado en 2007 que cedió en 2008 al caer nuevamente el número de trabajadores empleados en 2,806. Evidentemente, las enormes variaciones y la existencia de un gran número de trabajadores temporales reflejan el comportamiento de las ventas de

\footnotetext{
${ }^{6}$ Para incorporar el empleo del sector autopartista, se adicionó en la misma proporción cómo participan las compras nacionales para ensamble en el total de la proporción de la producción del sector autopartista (CIIU 343, de la EAM). Adicionalmente, se ajustó de acuerdo con la relación entre la producción bruta de la categoría I respecto de la producción bruta del CIIU 341.

40

| ISSN 1541-8561

FORUM EMPRESARIAL VOL. 19 NÚM. 1 • VERANO 2014
} 
vehículos, pues los mayores niveles de empleo corresponden a mayores ventas y viceversa, lo que obliga a calcular el empleo generado por vehículo producido.

Cuando se observa el total de personal remunerado por vehículo producido (Figura 6), la serie muestra nuevamente un comportamiento negativo.

Figura 6

Total personal remunerado por vehículo producido

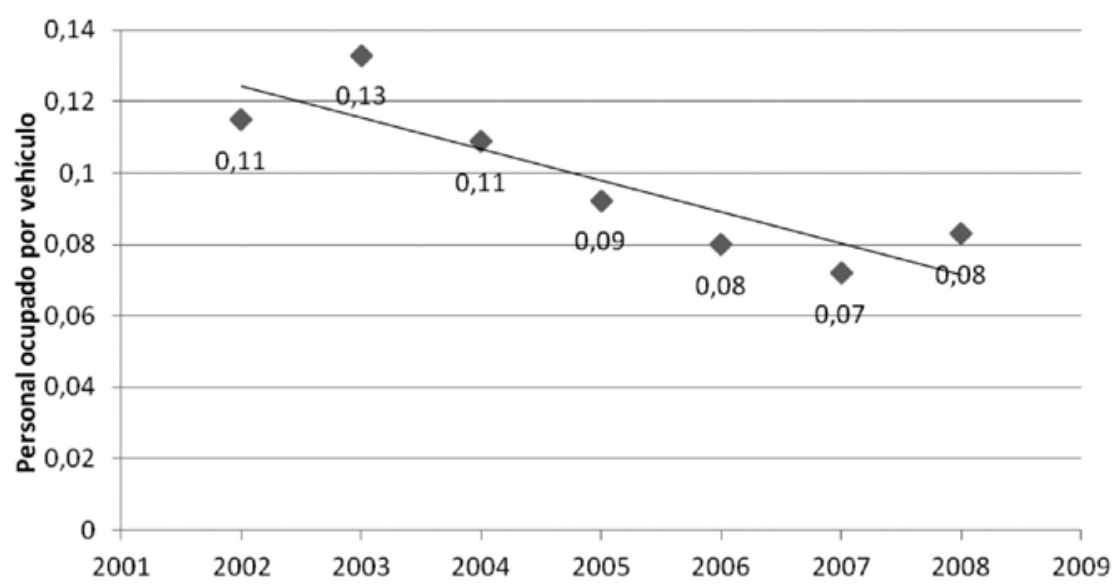

Fuente: DANE, ACOLFA (Manual estadístico), cálculos propios.

Esta menor cantidad de empleos por vehículos solo puede resultar o bien de un incremento en la productividad por incorporación tecnológica asociada a nuevas inversiones o de una menor participación de insumos nacionales en el valor total del vehículo. La información anterior y la depuración de las cifras (revaluación) muestran que, en efecto, los vehículos ensamblados utilizan de manera creciente más material no originario ( $\mathrm{MNO}$ por vehículo ensamblado) y haría falta mirar el comportamiento de la inversión en el sector de ensamble ya que ello sería un buen indicador para valorar la incorporación de nuevas tecnologías, ejercicio que se hace en la siguiente sección. 


\section{EFECTO SOBRE LA FORMACIÓN DE CAPITAL}

A partir de la Inversión neta, contenida en la Encuesta Anual Manufacturera preparada por el DANE, se presenta la evolución de la formación de capital bruto.

Las cifras muestran que tan solo durante los años 2004-2005 la inversión neta de la industria fue positiva, como se puede apreciar en la Figura 7. Para los demás años, ésta es negativa, lo que indica, por un lado, que el sector de ensamble de vehículos no ha contribuido a la formación bruta de capital, particularmente en los últimos años de la serie y, por otro, que no parecería que la disminución en el número de empleados por vehículo fabricado sea el resultado de crecientes inversiones en tecnología.

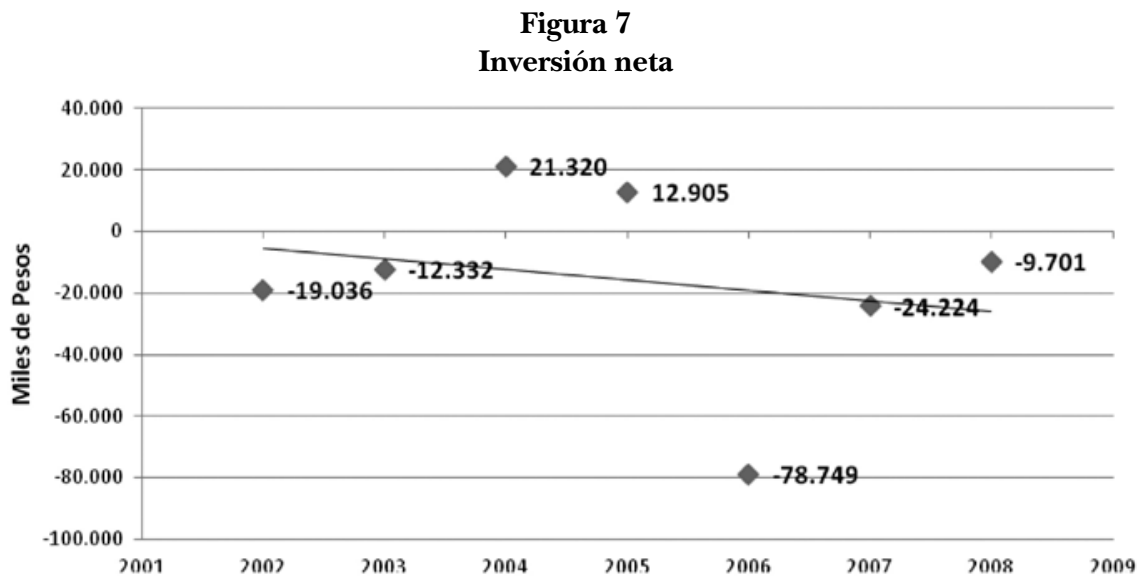

Fuente: Encuesta Nacional Manufacturera-DANE.

Nuevamente los datos de inversión generan duda respecto a la utilidad de la protección como mecanismo de promoción del crecimiento de la economía. En la siguiente sección se realiza un ejercicio que, precisamente, busca valorar el costo de la protección.

\section{COSTO DE LA PROTECCIÓN}

El costo de protección se obtiene de la siguiente forma:

$$
C P_{j t}=M N O_{j t} * n_{j t}+V A N_{j t} * t_{j}
$$


Donde:

$C P_{j t}=$ Costo de protección de la industria j en el año t. $M N O_{j t}=$ Sumatoria del CKD y la parte de origen extranjero del material originario utilizado por la industria j en el año t. $n_{j t}=$ Protección nominal de la industria j en el año t.

$V A N_{j t}=$ Sumatoria del valor agregado más la parte de origen nacional del material originario para la industria j en el año t. $t_{j t}=$ Arancel a las importaciones de los bienes terminados de la industria j en el año t.

Lo que busca la anterior formulación es calcular cuánto cuestan las exenciones arancelarias que el estado le otorga a la industria de ensamblaje y de autopartes sumado al costo que paga el consumidor por la protección a la incorporación de valor agregado nacional.

Tabla 5

Costo de Protección

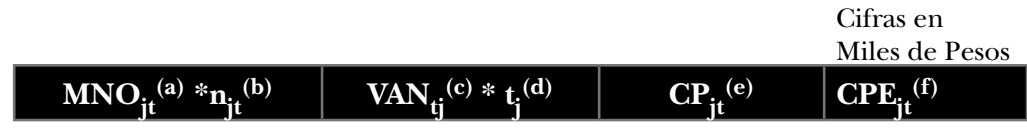

\begin{tabular}{|c|c|c|c|c|}
\hline 2002 & 388.537 .117 & 182.658 .099 & 571.195 .216 & 88.902 \\
\hline 2003 & 368.285 .314 & 167.071 .162 & 535.356 .476 & 91.577 \\
\hline 2004 & 589.355 .003 & 291.407 .402 & 880.762 .405 & 126.765 \\
\hline 2005 & 658.527 .606 & 334.418 .561 & 992.946 .167 & 141.627 \\
\hline 2006 & 818.975 .301 & 421.051 .268 & 1.240 .026 .570 & 146.230 \\
\hline 2007 & 1.027 .039 .034 & 556.481 .287 & 1.583 .520 .321 & 151.795 \\
\hline 2008 & 534.462 .162 & 324.527 .555 & 858.989 .717 & 112.640 \\
\hline
\end{tabular}

Fuente: Ministerio de Comercio, Industria y Turismo, DANE, cálculos propios Notas:

(a) Valor del CKD más la parte de origen extranjero del material originario.

(b) Protección nominal tomada de la Figura 1.

(c) Valor del valor agregado por la industria más la parte de origen nacional del material originario.

(d) Arancel a la importación de vehículos de la categoría I.

(e) Sumatoria de las dos columnas anteriores.

(f) Costo de la protección por empleado, que se tomó del número de empleados que aparecen reflejados en la Figura 5.

\begin{tabular}{l|l} 
ISSN $1541-8561$ & 43
\end{tabular} 
Lo que refleja la Tabla 5 es el costo que para la sociedad colombiana (Estado y consumidores) representa la generación de cada empleo en el sector de ensamble y producción de autopartes como resultado de la protección vigente por el régimen automotriz especial de la Comunidad Andina.

Los materiales no originarios, tanto de los autopartistas como de los ensambladores, entran al país sin arancel y el resultado final es un vehículo que se vende al público con el beneficio de precio que resulta de un mercado que tiene un sobreprecio del $35 \%$ producto del arancel. El valor de insumos y agregado nacional incorporado al vehículo también goza del beneficio de la protección. La Tabla 5 calcula, en consecuencia, los valores agregados nacionales y los materiales no originarios y los multiplica por la protección $(35 \%)$ para arrojar un Costo de protección (CP) que el régimen automotriz impone al Estado y los consumidores en la producción de los vehículos de la categoría 1. Este valor alcanzó un pico en 2007 con 1.5 billones de pesos. Este costo lo asume, en parte, el fisco por las exenciones tributarias sobre los insumos importados y, en parte, el consumidor por el mayor precio que paga en el mercado por los vehículos ensamblados para subsidiar la incorporación de valor agregado nacional. Este cálculo no se hace para los vehículos importados ya que si bien el consumidor sí paga un sobreprecio, este sobreprecio (arancel) entra a las arcas del Estado que lo revierte a la sociedad en forma de gasto público.

La última columna de la Tabla 5 divide el costo de la protección por el número total de empleados a los que se refiere la Gráfica 5, es decir, los permanentes, los temporales y lo que se genera durante el proceso de ensamble en razón a la demanda de autopartes, lo que permite valorar cuánto le cuesta al país la generación de cada empleo. Las cifras empiezan en 2002 con un costo por empleo de cerca de 89 millones de pesos año, y crece a 151 millones de pesos año por empleo generado en 2007, presentándose una ligera caída en este costo en 2008. 


\section{Reflexiones finales}

Los datos que arroja la presente investigación deben provocar una profunda reflexión respecto a la realidad del mercado automotor en Colombia, el cual requiere con urgencia una revisión. Se deben tener en consideración los siguientes aspectos:

- Protección creciente y prolongada.

- Baja incorporación de insumos originarios del país.

- Bajo impacto de la protección sobre empleo e inversión.

- Alto costo de la protección y alto costo en la generación de empleo.

El nuevo ambiente de apertura en Colombia está dado por la suscripción de libre comercio, pero a la fecha los plazos de desgravación que se han negociado con Estados Unidos, Europa y Corea establecen plazos largos (8-10 años), lo que hace que no sean estos tratados los instrumentos que ayuden a resolver, en un corto plazo, las ineficiencias del sistema que son evidentes en el presente trabajo. El país y los consumidores seguirán pagando subsidios de manera decreciente a las empresas ensambladoras y autopartistas que desaparecerán cuando se surta la desgravación pactada en los acuerdos y en ese momento la industria local tendrá que haber adquirido los niveles de eficiencia que requiere la competencia internacional, como sucedió con la apertura en Suráfrica, o desaparecerá, como sucedió en Chile. 


\section{RefERenCias}

Alavi, R. (1996). Industrialization in Malaysia: import substitution and infant industry perfomance. London: Routledge.

Balassa, B. (1965). Tariff protection in industrial countries: An evaluation. The Journal of Political Economy, 73(6), 573-594.

Basevi, G. (1966). The United States tariff structure: estimates of effective rates of protection of United States industries and industrial labor. The Review of Economics and Statistics, 48(2), 147160.

Cohen, B. I. (1971). The use of effective tariffs. The Journal of Political Economy, 79(1), 128-141.

Echavarría, J. J., Villamizar, M. y González, J. (2006). El proceso colombiano de desindustrialización. Borradores de Economía No.361. Colombia: Banco de la República.

Farné, S. (2008). Las Cooperativas de Trabajo Asociado en Colombia: balance de la política gubernamental 2002-2007. Revista de Economía Institucional 10(18), 261-285.

Grubel, H. G. y Johnson, H. G. (1967). Nominal tariffs, indirect taxes and effective rates of protection: The common market. The Economic Journal, 77(308), 761-776.

Prebisch, R. (1964). Towards a new trade policy for development. En G. Meier, Leading Issues in Economic Development (20-26). Londres: Oxford University Press. 


\section{Anexo 1}

Industria automotriz colombiana 2002 - 2008 - Categoría I

Producción bruta, consumo intermedio y valor agregado

Valores corrientes

\begin{tabular}{|c|c|c|c|c|c|}
\hline & & & & & \multirow{2}{*}{$\begin{array}{l}\text { Cifras en } \\
\text { miles de Pesos } \\
\text { Valor } \\
\text { agregado(b) }\end{array}$} \\
\hline & $\begin{array}{l}\text { Producción } \\
\text { bruta(a) }\end{array}$ & \multicolumn{3}{|c|}{ Consumo Intermedio } & \\
\hline & & CKD (c) & $\mathrm{MO}_{1}(\mathrm{~d})$ & $\mathrm{MO}_{2}(\mathrm{e})$ & \\
\hline 2002 & 1.680 .275 .676 & 954.948 .974 & 203.446 .418 & 190.395 .933 & 331.484 .350 \\
\hline 2003 & 1.573 .165 .741 & 904.643 .856 & 191.175 .707 & 186.901 .096 & 290.445.082 \\
\hline 2004 & 2.585 .658 .667 & 1.414 .196 .335 & 338.869 .754 & 284.666 .530 & 547.926 .048 \\
\hline 2005 & 2.916.213.605 & 1.543 .856 .812 & 416.875 .190 & 344.111 .569 & 611.370 .033 \\
\hline 2006 & 3.637 .940 .159 & 1.875 .480 .401 & 559.456 .134 & 355.364 .604 & 847.639 .020 \\
\hline 2007 & 4.647.012.233 & 2.279 .977 .540 & 777.088 .159 & 404.445 .368 & 1.185 .501 .167 \\
\hline 2008 & 2.515 .446 .105 & 1.209 .995 .920 & 378.228 .598 & 266.012.267 & 661.209 .320 \\
\hline
\end{tabular}

Fuente: Ministerio de Comercio, Industria y Turismo, DANE, cálculos propios.

Notas:

(a) La producción bruta para la categoría 1 se calculó a partir de los datos del consumo intermedio y el valor agregado del CIIU 341 de la EAM.

(b) El valor agregado se calculó como la proporción de la producción bruta.

(c) El CKD o Material No Originario se tomó del reporte de las ensambladoras de vehículos.

(d) El Material Originario no originario se obtuvo a partir de determinar el porcentaje del componente importado del CIIU 343.

(e) El Material Originario se tomó del reporte de las ensambladoras vehículos, sustrayendo el Material Originario no originario. 


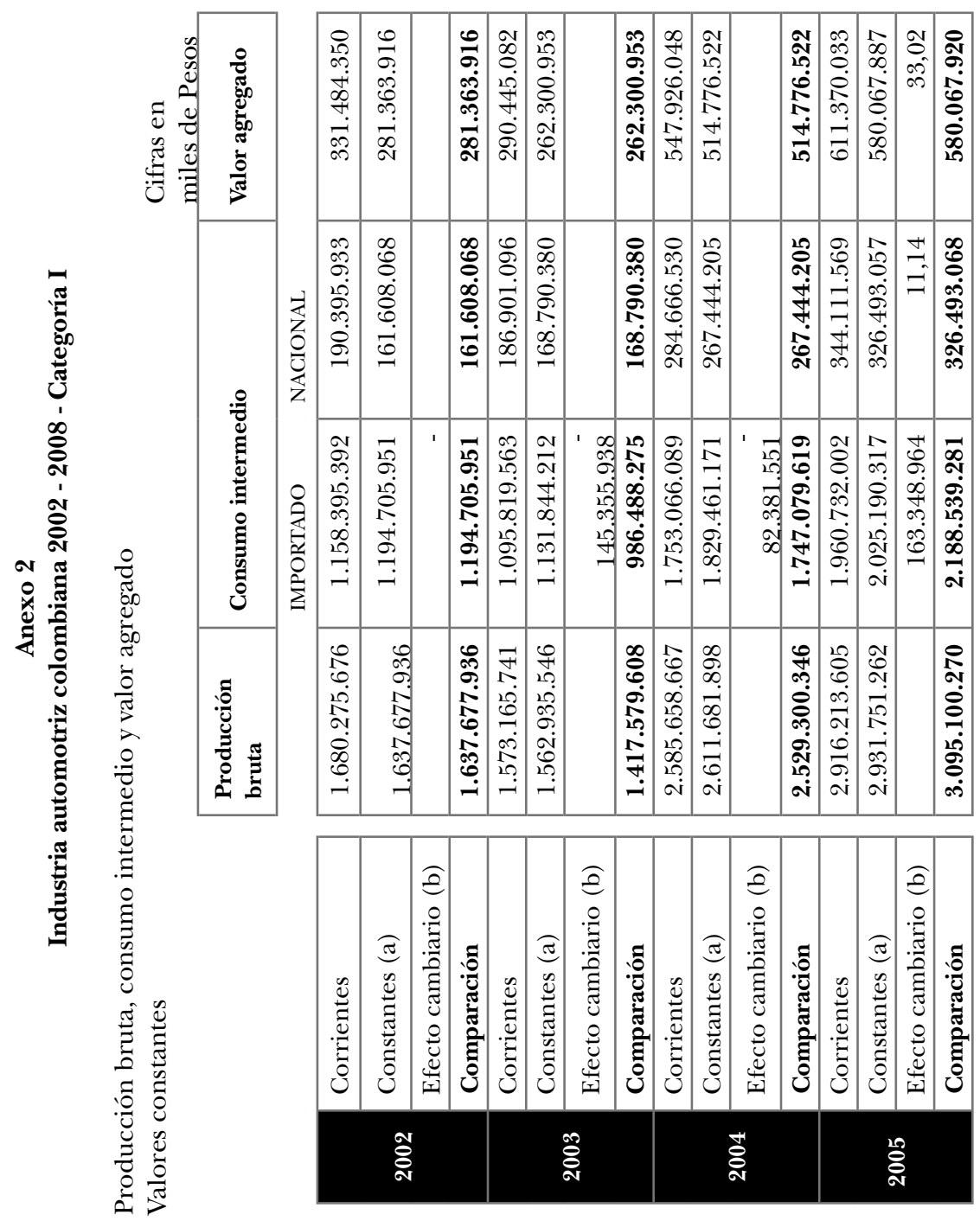




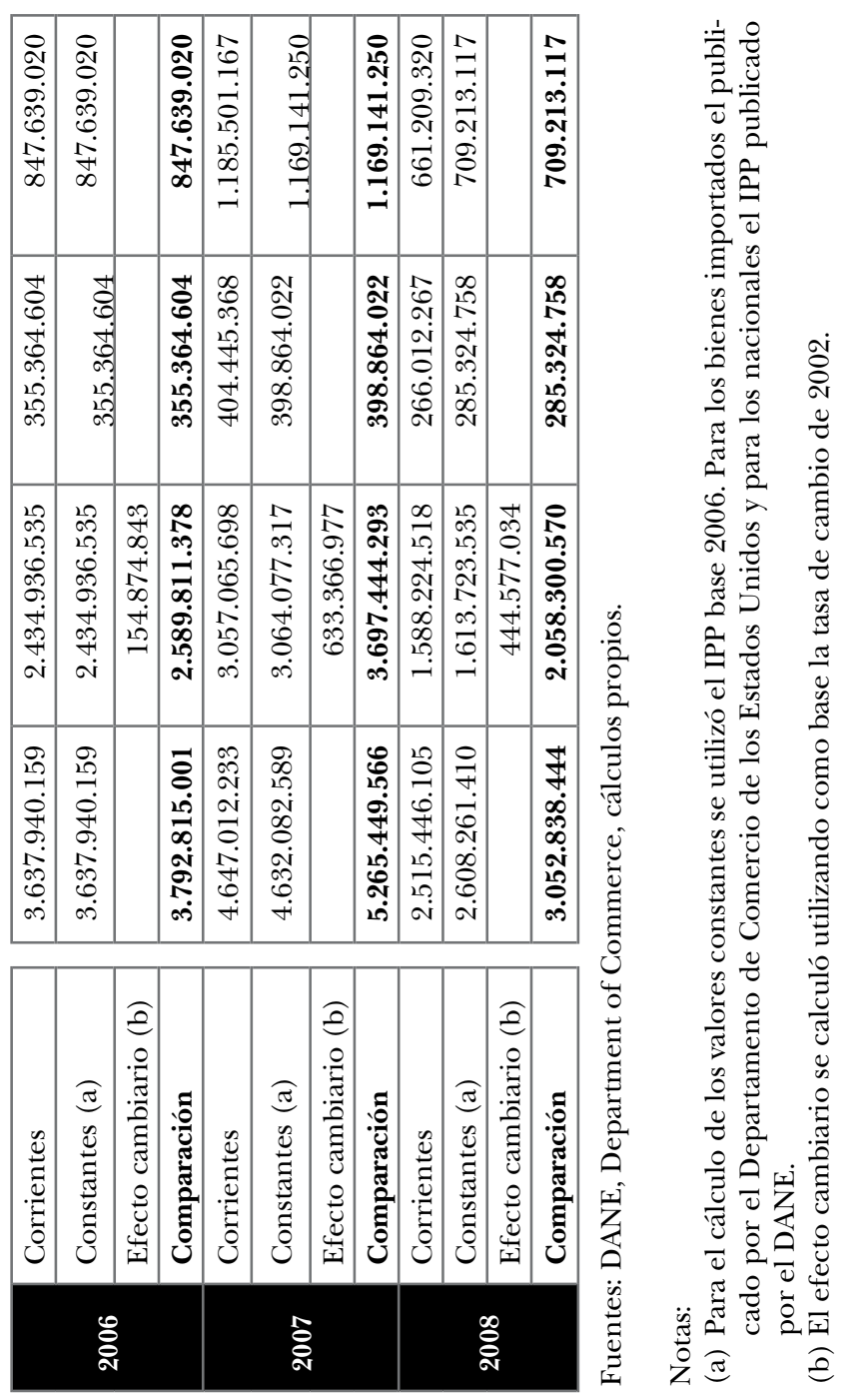


Anexo 3

Industria automotriz colombiana 2002 - 2008 - Categoría I

Producción bruta, consumo intermedio y valor agregado

Cifras en

miles de Pesos

\begin{tabular}{|r|r|r|}
\hline \multicolumn{1}{|c}{ PB } & \multicolumn{1}{c|}{ aij } & Vj \\
\hline \multicolumn{3}{|c|}{} \\
\hline 1.637 .677 .936 & 1.194 .705 .951 & 161.608 .068 \\
\hline $100,00 \%$ & $72,95 \%$ & $11,92 \%$ \\
\hline 1.417 .579 .608 & 986.488 .275 & 168.790 .380 \\
\hline $100,00 \%$ & $69,59 \%$ & $14,61 \%$ \\
\hline 2.529 .300 .346 & 1.747 .079 .619 & 267.444 .205 \\
\hline $100,00 \%$ & $69,07 \%$ & $13,28 \%$ \\
\hline 3.095 .100 .270 & 2.188 .539 .281 & 326.493 .068 \\
\hline $100,00 \%$ & $70,71 \%$ & $12,98 \%$ \\
\hline 3.792 .815 .001 & 2.589 .811 .378 & 355.364 .604 \\
\hline $100,00 \%$ & $68,28 \%$ & $12,07 \%$ \\
\hline 5.265 .449 .566 & 3.697 .444 .293 & 398.864 .022 \\
\hline $100,00 \%$ & $70,22 \%$ & $9,74 \%$ \\
\hline 3.052 .838 .444 & 2.058 .300 .570 & 285.324 .758 \\
\hline $100,00 \%$ & $67,42 \%$ & $12,17 \%$ \\
\hline
\end{tabular}

Fuente: cálculos propios.

Notas: vj se calcula con base en la resolución 323. 
Proteccionismo en LA INDUSTRIA AUtOMOtRIZ COLOMBIANA
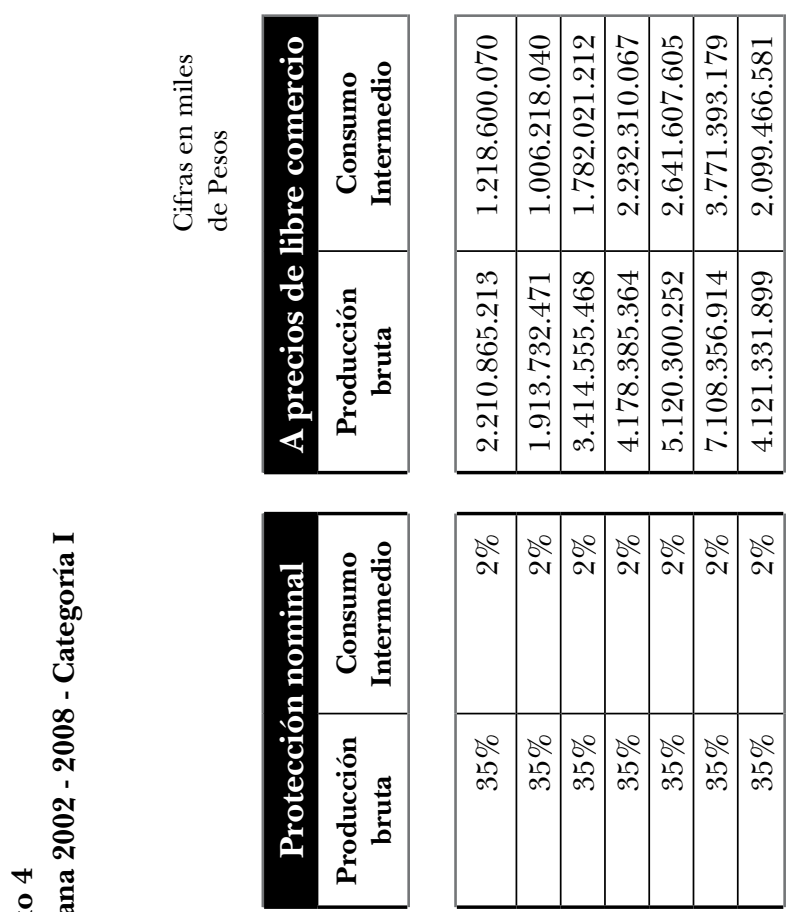

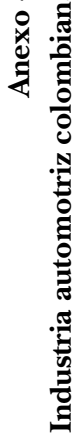

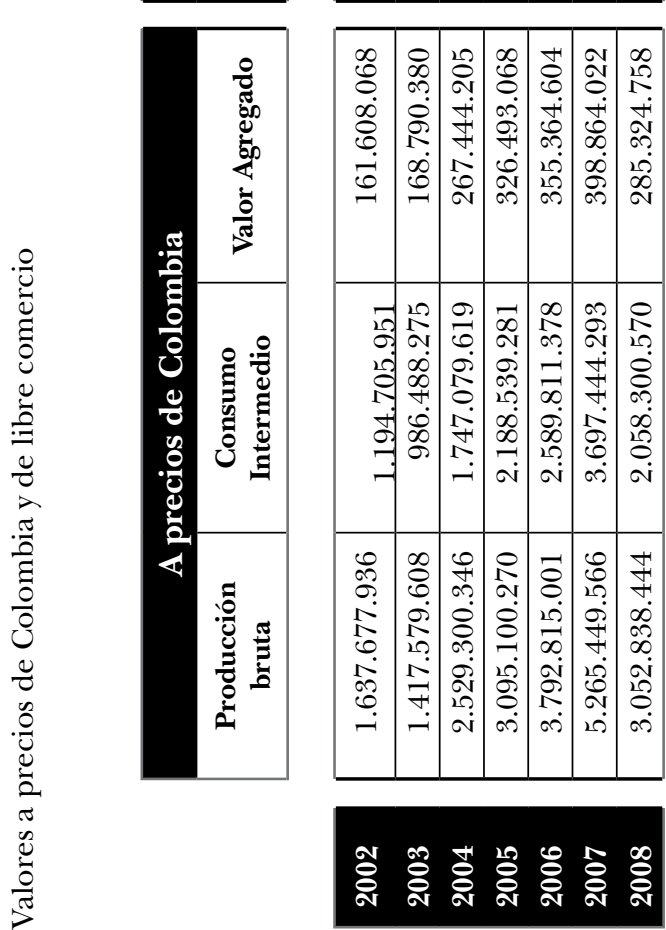

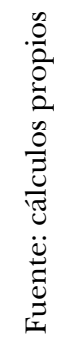


Anexo 5

Por qué se excluyeron las cifras de 2009

1. La participación de MO, según cifras del Ministerio de Comercio, creció $1,34 \%$ en 2008 y tan solo un $0,27 \%$ en 2009 , no obstante, la cifras de ENM muestran que el valor agregado de la industria ensambladora (CIIU 341) creció 2,21\% en 2008 y 0,77\% en 2009.

2. La participación de CKD por vehículo producido, eliminando el efecto de la tasa de cambio, fue para 2008 de US $\$ 8.077$, mientras que para el 2009 esta cifra fue de US $\$ 4.944$, lo que resulta incoherente a la luz de las cifras de incorporación reportadas ante el Ministerio.

3. Otro tanto sucede con el MO cuando se mide por vehículo, ya que en 2008 el MO por vehículo fue de Col. \$6.629.000, mientras que para 2009 fue de Col. $\$ 4.938 .000$.

Los aspectos mencionados en los puntos anteriores muestran enormes inconsistencias dado que no es dable que baje simultáneamente la participación de $\mathrm{MO}$ y $\mathrm{MNO}$. El único escenario en que esto sería posible es ante una baja en los CKD, pero revisados éstos en la información de los manifiestos de importación (Legiscomex), no se encontró un menor precio unitario. Las razones expuestas llevaron a no incluir a 2009 en la serie en la cual se calculó la protección efectiva.

CKD y MO por vehículo

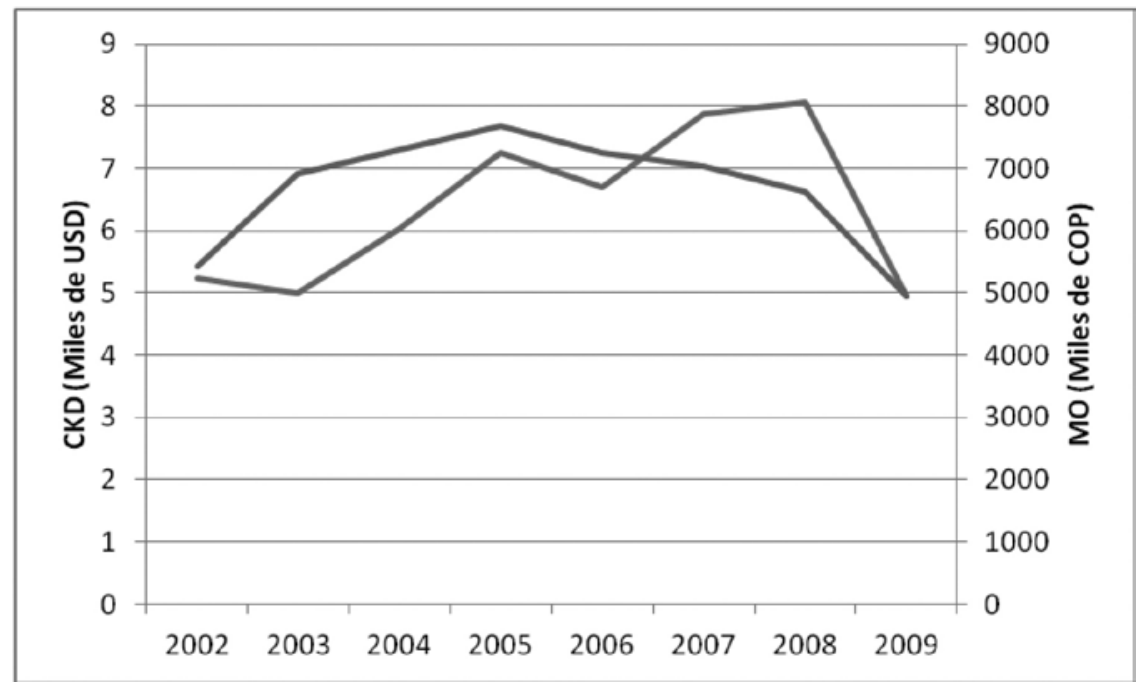

